\title{
Partial characterization of graphs having a single large Laplacian eigenvalue
}

\author{
L. Emilio Allem*\| Antonio Cafure ${ }^{\dagger \dagger} \|$ Ezequiel Dratman ${ }^{\dagger \S \|}$ \\ Luciano N. Grippo ${ }^{\S \|}$ Martín D. Safe $\mathbb{q}^{\|}$Vilmar Trevisan*\|
}

Submitted: Sep 19, 2017; Accepted: Nov 1, 2018; Published: Dec 21, 2018

(C) The authors. Released under the CC BY-ND license (International 4.0).

\begin{abstract}
The parameter $\sigma(G)$ of a graph $G$ stands for the number of Laplacian eigenvalues greater than or equal to the average degree of $G$. In this work, we address the problem of characterizing those graphs $G$ having $\sigma(G)=1$. Our conjecture is that these graphs are stars plus a (possible empty) set of isolated vertices. We establish a link between $\sigma(G)$ and the number of anticomponents of $G$. As a by-product, we present some results which support the conjecture, by restricting our analysis to cographs, forests, and split graphs.
\end{abstract}

Mathematics Subject Classifications: 05C50; 97K30; 35Pxx

\section{Introduction}

Let $G$ be a graph on $n$ vertices and $m$ edges and let $d_{1} \geqslant \cdots \geqslant d_{n}$ be its degree sequence. We denote by $A(G)$ its adjacency matrix and by $D(G)$ the diagonal matrix having $d_{i}$ in the diagonal entry $(i, i)$, for every $1 \leqslant i \leqslant n$, and 0 otherwise. The Laplacian matrix of $G$ is the positive semidefinite matrix $L(G)=D(G)-A(G)$. The eigenvalues of $L(G)$ are called Laplacian eigenvalues of $G$; the spectrum of $L(G)$ is the Laplacian spectrum of $G$ and will be denoted by $L \operatorname{spec}(G)$. Since it is easily seen that 0 is a Laplacian eigenvalue and it is well-known that Laplacian eigenvalues are less than or equal to $n$ it turns out

*Instituto de Matemática, Universidade Federal do Rio Grande do Sul, Brazil

†CONICET, Argentina

${ }^{\ddagger}$ Instituto del Desarrollo Humano, Universidad Nacional de General Sarmiento, Argentina.

Depto. de Matemática, CBC, Universidad de Buenos Aires, Argentina

$\S$ Instituto de Ciencias, Universidad Nacional de General Sarmiento, Argentina

`Departamento de Matemática, Universidad Nacional del Sur, Argentina.

INMABB, Universidad Nacional del Sur (UNS)-CONICET, Argentina

"IEmail addresses: emilio.allem@ufrgs.br (L. E. Allem), acafure@ungs.edu.ar (A. Cafure), edratman@ungs.edu.ar (E. Dratman), lgrippo@ungs.edu.ar (L. N. Grippo),

msafe@uns.edu.ar (M. D. Safe), trevisan@mat.ufrgs.br (V. Trevisan) 
that $\operatorname{Lspec}(G) \subset[0, n]$. From now on, if $\operatorname{Lspec}(G)=\left\{\mu_{1}, \mu_{2}, \ldots, \mu_{n}\right\}$, we will assume that $\mu_{1} \geqslant \mu_{2} \geqslant \cdots \geqslant \mu_{n}$, where $\mu_{n}=0$.

Understanding the distribution of Laplacian eigenvalues of graphs is a problem that is both relevant and difficult. It is relevant due to the many applications related to Laplacian matrices (see, for example, $[9,10]$ ). It seems to be difficult because little is known about how the Laplacian eigenvalues are distributed in the interval $[0, n]$.

Our main motivation is understanding the structure of graphs that have few large Laplacian eigenvalues. In particular, we would like to characterize graphs that have a single large Laplacian eigenvalue. What do we mean by a large Laplacian eigenvalue? A reasonable measure is to compare this eigenvalue with the average of all eigenvalues. Since the average of Laplacian eigenvalues equals the average degree $\bar{d}(G)=\frac{2 m}{n}$ of $G$, we say that a Laplacian eigenvalue is large if it is greater than or equal to the average degree.

Inspired by this idea, the paper [3] introduces the spectral parameter $\sigma(G)$ which counts the number of Laplacian eigenvalues greater than or equal to $\bar{d}(G)$. Equivalently, $\sigma(G)$ is the largest index $i$ for which $\mu_{i} \geqslant \frac{2 m}{n}$. Since the greatest Laplacian eigenvalue $\mu_{1}$ is at least $\frac{2 m}{n}$ then it follows that $\sigma(G) \geqslant 1$.

There is evidence that $\sigma(G)$ plays an important role in defining structural properties of a graph $G$. For example, it is related to the clique number $\omega$ of $G$ (the number of vertices of the largest induced complete subgraph of $G$ ) and it also gives insight about the Laplacian energy of a graph [11,3]. Pirzada and Ganie [11] conjectured that $\sigma(G) \geqslant \omega-1$. Later, this conjectured was disproved in [3] by showing a counterexample within the class of threshold graphs. Moreover, several structural properties of a graph are related to $\sigma$ (see, for example, $[2,3]$ ).

In this paper we are concerned with furthering the study of $\sigma(G)$. In particular, we deal with a problem posed in [3] which asks for characterizing all graphs $G$ having $\sigma(G)=1$; i.e., having only one large Laplacian eigenvalue. Our conjecture is that the only connected graph on $n$ vertices having $\sigma=1$ is the star $K_{1, n-1}$ and that the only disconnected graph on $n$ vertices having $\sigma=1$ is a star together with some isolated vertices. More precisely, we conjecture that graphs having $\sigma=1$ are some stars plus a (possibly empty) set of isolated vertices. From now on, $K_{1, r}+s K_{1}$ denotes the star on $r+1$ vertices plus $s$ isolated vertices.

Conjecture 1. Let $G$ be a graph. Then $\sigma(G)=1$ if and only if $G$ is isomorphic to $K_{1}$, $K_{2}+s K_{1}$ for some $s \geqslant 0$, or $K_{1, r}+s K_{1}$ for some $r \geqslant 2$ and $0 \leqslant s<r-1$.

In this work, we show that this conjecture is true if it holds for graphs which are simultaneously connected and co-connected (Conjecture 11) and prove that Conjecture 1 is true for cographs, forests, and split graphs. The main tool for proving our results is an interesting link we have found between $\sigma$ and the number of anticomponents of $G$ (see Section 2). The interesting feature of this result is that it relates a spectral parameter with a classical structural parameter. Studying structural properties of the anticomponents of $G$ may shed light on the distribution of Laplacian eigenvalues and, reciprocally, the distribution of Laplacian eigenvalues should give insight about the structure of the graph. 
This article is organized as follows. In Section 2 we state definitions and previous results concerning Laplacian eigenvalues. In Section 3, we present some new results which establish the connection between $\sigma$ and the number of nonempty anticomponents of $G$. In Section 4, we present some evidence on the validity of Conjecture 1 by proving that the conjecture is true when $G$ is a cograph, a forest, or a split graph.

\section{Definitions}

In this article, all graphs are finite, undirected, and without multiple edges or loops. All definitions and concepts not introduced here can be found in [13]. We say that a graph is empty if it has no edges. A trivial graph is a graph with precisely one vertex; every trivial graph is isomorphic to the graph which we will denote by $K_{1}$. A graph is nontrivial if it has more than one vertex.

We use the standard notation $\Delta(G)$ to denote the maximum degree of a graph $G$.

Let $G_{1}$ and $G_{2}$ be two graphs such that $V\left(G_{1}\right) \cap V\left(G_{2}\right)=\emptyset$. The disjoint union of $G_{1}$ and $G_{2}$, denoted $G_{1}+G_{2}$, is the graph whose vertex set is $V\left(G_{1}\right) \cup V\left(G_{2}\right)$, and its edge set is $E\left(G_{1}\right) \cup E\left(G_{2}\right)$. We write $k G$ to represent the disjoint union $G+\cdots+G$ of $k$ copies of a graph $G$. The join of $G_{1}$ and $G_{2}$, denoted $G_{1} \vee G_{2}$, is the graph obtained from $G_{1}+G_{2}$ by adding new edges from each vertex of $G_{1}$ to every vertex of $G_{2}$.

A vertex $v$ of a graph $G$ is a twin of another vertex $w$ of $G$ if they both have the same neighbors in $V(G) \backslash\{v, w\}$. We say that a graph $G^{\prime}$ is obtained from $G$ by adding a twin $v^{\prime}$ to a vertex $v$ of $G$ if $V\left(G^{\prime}\right)=V(G) \cup\left\{v^{\prime}\right\}, v^{\prime}$ is a twin of $v$ in $G^{\prime}$, and $G^{\prime}-v^{\prime}$ is isomorphic to $G$.

By $G[S]$ we denote the subgraph of $G$ induced by a subset $S \subseteq V(G)$.

We use $\bar{G}$ to denote the complement graph of a graph $G$. An anticomponent of a graph $G$ is the subgraph of $G$ induced by the vertex set of a connected component of $\bar{G}$. More precisely, an induced subgraph $H$ of $G$ is an anticomponent if $\bar{H}$ is a connected component of $\bar{G}$. Notice that if $G_{1}, G_{2}, \ldots, G_{k}$ are the anticomponents of $G$, then $G=G_{1} \vee \cdots \vee G_{k}$. A graph $G$ is co-connected if $\bar{G}$ is connected.

A forest is a graph with no cycles and a tree is a connected forest. The complete graph on $n$ vertices is denoted by $K_{n}$. A universal vertex of a graph $G$ is a vertex $v$ adjacent to every vertex $w$ different from $v$. A star is a graph isomorphic to $K_{1}$ or to a tree with a universal vertex. We use $K_{1, n-1}$ to denote the star on $n$ vertices, where $K_{1,0}$ is isomorphic to $K_{1}$ and $K_{1,1}$ is isomorphic to $K_{2}$. The chordless path (respectively, cycle) on $k$ vertices is denoted by $P_{k}$ (respectively, $C_{k}$ ).

A stable set of a graph is a set of pairwise nonadjacent vertices. A clique of a graph is a set of pairwise adjacent vertices.

Throughout this article, given two graphs $G$ and $H$, we write $G=H$ to point out that $G$ and $H$ belong to the same isomorphism class.

Brouwer and Haemers [1] provided a lower bound for the $k$-th Laplacian eigenvalue of a graph in terms of $d_{k}$, answering a conjecture raised by Guo [6].

Theorem 2 ([1]). Let $G$ be a graph on $n$ vertices. If $G$ is not isomorphic to $K_{k}+(n-k) K_{1}$, then $\mu_{k}(G) \geqslant d_{k}-k+2$. 
Throughout this article we will use the lower bounds corresponding to the cases $k=$ $1[5]$ and $k=2[7]$.

It is easy to prove that the Laplacian spectrum of the disjoint union $G_{1}+G_{2}$ is the union of the Laplacian spectrums of $G_{1}$ and $G_{2}$. The next result allows to determine the Laplacian spectrum of the join $G_{1} \vee G_{2}$, from those of $G_{1}$ and $G_{2}$.

Theorem 3 ([8, Theorem 2.20]). Let $G_{1}$ and $G_{2}$ be two graphs with Laplacian spectrums $\mu_{1} \geqslant \mu_{2} \geqslant \cdots \geqslant \mu_{n_{1}}=0$ and $\lambda_{1} \geqslant \lambda_{2} \geqslant \cdots \geqslant \lambda_{n_{2}}=0$, respectively. Then the Laplacian eigenvalues of $G_{1} \vee G_{2}$ are $n_{1}+n_{2} ; n_{2}+\mu_{i}$, for $1 \leqslant i \leqslant n_{1}-1 ; n_{1}+\lambda_{i}$, for $1 \leqslant i \leqslant n_{2}-1$ and 0.

\section{Relating $\sigma$ and the number of anticomponents}

This section is devoted to establish a link between $\sigma(G)$ and the number of anticomponents of $G$.

In virtue of Theorem 3 , the following result immediately holds.

Lemma 4. If $G=G_{1} \vee \cdots \vee G_{k}$, with $k \geqslant 1$, is a graph on $n$ vertices, then $n$ is a Laplacian eigenvalue of $G$ with multiplicity at least $k-1$.

Lemma 5. If $G$ has $k$ anticomponents, then $k \leqslant \sigma(G)+1$.

Proof. Let $G=G_{1} \vee \cdots \vee G_{k}$ where $G_{1}, \ldots, G_{k}$ are the anticomponents of $G$. For any graph $G$ with at least one vertex we have that $\sigma(G) \geqslant 1$ and thus the assertion follows when $k=1$. We may assume that $k \geqslant 2$. Lemma 4 implies that $n$ is a Laplacian eigenvalue of $G$ with multiplicity at least $k-1$ in $G$. Thus $\mu_{k-1}(G)=n$ which implies that $\sigma(G) \geqslant k-1$.

Remark 6 . The upper bound given by Lemma 5 is sharp when $\sigma(G)>1$. Indeed, for $s \geqslant 2$ consider the graph $G=4 K_{2} \vee K_{1} \vee \cdots \vee K_{1}$, where $s$ is the number of $K_{1}$ 's. The average degree of $G$ is $s+7-\frac{48}{s+8}$ and it has $s+1$ anticomponents. Since its Laplacian eigenvalues are $s+8, s+2, s$, and 0 with multiplicities $s, 4,3$, and 1 , respectively, it follows that $\sigma(G)=s$.

We use $\ell(G)$ to denote the number of nonempty anticomponents of a graph $G$. Recall that a nontrivial graph has at least two vertices. The following result looks further into the case where equality holds in Lemma 5 showing that $\sigma(G)$ is an upper bound for $\ell(G)$.

Theorem 7. Let $G$ be a graph having $k=\sigma(G)+1$ anticomponents. Then $\ell(G) \leqslant \sigma(G)$. Moreover, if $\sigma(G)=\ell(G)$, then the remaining anticomponent of $G$ is empty but nontrivial.

Proof. Write $G=G_{1} \vee \cdots \vee G_{k}$ where $G_{1}, \ldots, G_{k}$ are the anticomponents of $G$. Since $\sigma(G) \geqslant 1$ then $k \geqslant 2$. We set the following notations for each $i \in\{1, \ldots, k\}$ :

$$
n_{i}=\left|V\left(G_{i}\right)\right|, \quad m_{i}=\left|E\left(G_{i}\right)\right|, \quad \mu_{1}^{(i)}=\mu_{1}\left(G_{i}\right) .
$$


Assume that $G_{1}, \ldots, G_{\ell}$ are the nonempty anticomponents. Since $k \geqslant 2$ and we are assuming that $\sigma(G)=k-1$ it turns out that $\mu_{k}(G)<\bar{d}(G)$. Therefore, for each $i \in$ $\{1, \ldots, k\}$ such that $n_{i}>1$ we have that

$$
n-n_{i}+\mu_{1}^{(i)} \leqslant \mu_{k}(G)<\frac{2 m}{n}=\frac{2 \sum_{j=1}^{k} m_{j}+2 \sum_{1 \leqslant i<j \leqslant k} n_{i} n_{j}}{n},
$$

the first inequality holds by Theorem 3. Equivalently,

$$
\begin{aligned}
\mu_{1}^{(i)} & <\frac{2 \sum_{j=1}^{k} m_{j}-\left(n^{2}-2 \sum_{1 \leqslant i<j \leqslant k} n_{i} n_{j}\right)}{n}+n_{i} \\
& =\frac{2 \sum_{j=1}^{k} m_{j}-\sum_{j=1}^{k} n_{j}^{2}+n n_{i}}{n} .
\end{aligned}
$$

As a consequence of Theorem 2, we obtain the following lower bound for each $i \in$ $\{1, \ldots, \ell\}$ :

$$
\mu_{1}^{(i)} \geqslant \Delta\left(G_{i}\right)+1 \geqslant \bar{d}\left(G_{i}\right)+1=\frac{2 m_{i}}{n_{i}}+1 .
$$

Combining (1) and (2), we deduce that, for each $i \in\{1, \ldots, \ell\}$,

$$
2 n_{i} \sum_{j=1}^{k} m_{j}-n_{i} \sum_{j=1}^{k} n_{j}^{2}+n n_{i}^{2}-2 n m_{i}-n n_{i}>0 .
$$

Arguing towards a contradiction, suppose that $\ell(G)=k$. If we sum up the left-hand side of (3) for each $i \in\{1, \ldots, k\}$, we obtain

$$
2 n \sum_{j=1}^{k} m_{j}-n \sum_{j=1}^{k} n_{j}^{2}+n \sum_{i=1}^{k} n_{i}^{2}-2 n \sum_{i=1}^{k} m_{i}-n^{2}=-n^{2}
$$

which is not a positive quantity. This contradiction proves that $G$ has at most $k-1=\sigma(G)$ nonempty anticomponents and our first assertion follows.

Assume now that $\ell(G)=k-1$. Suppose that $G_{k}$ is trivial. Hence $n_{k}=1$ and $m_{k}=0$. Summing up to the left-hand side of (3) for each $i \in\{1, \ldots, k-1\}$, we obtain that

$$
-2 \sum_{j=1}^{k-1} m_{j}+\sum_{j=1}^{k} n_{j}^{2}-n^{2}=-2 \sum_{j=1}^{k-1} m_{j}-2 \sum_{1 \leqslant i<j \leqslant k} n_{i} n_{j}
$$

should be a positive number. This contradiction shows that $G_{k}$ must be nontrivial.

Recall that a bipartite graph is a graph whose set of vertices can be partitioned into two (possibly empty) stable sets called partite sets of the bipartite graph. A complete bipartite graph is a bipartite graph isomorphic to $r K_{1} \vee s K_{1}$ for two positive integers $r$ and $s$. We denote by $K_{r, s}$ the complete bipartite graph isomorphic to $r K_{1} \vee s K_{1}$. The upper bound $\sigma(G)$ on $\ell(G)$ for those graphs having exactly $\sigma(G)+1$ anticomponents is not tight when $\sigma(G)=1$. Indeed, the following result shows that if a graph $G$ has $\sigma(G)=1$, then $G$ has no nonempty anticomponents. 
Corollary 8. If $G$ is a graph on $n$ vertices with $\sigma(G)=1$ and $\bar{G}$ is disconnected, then $G=K_{1, n-1}$.

Proof. In virtue of Lemma 5, the number of anticomponents of $G$ is at most 2. Since $\bar{G}$ is disconnected, we conclude that $G$ has precisely two anticomponents $G_{1}$ and $G_{2}$ and thus $G=G_{1} \vee G_{2}$.

Suppose, for a contradiction, that $G_{1}$ is a nonempty anticomponent of $G$. Because of Theorem 7 , we conclude that $G_{2}$ is empty but nontrivial. Following the notation used in the proof of Theorem 7 , we have that $m_{2}=0$. For $i=1$, inequality (3) becomes

$$
-2 n_{2} m_{1}-n_{1} n_{2}^{2}+n_{2} n_{1}^{2}-n_{1}^{2}-n_{1} n_{2}>0 .
$$

Since $G_{2}$ is a nontrivial empty graph it follows that $\mu_{1}^{(2)}=0$ and hence, for $i=2$, inequality (1) becomes

$$
2 m_{1}-n_{1}^{2}+n_{1} n_{2}>0 .
$$

Summing up (4) and $n_{2}$ times (5) gives

$$
-n_{1}^{2}-n_{1} n_{2}>0
$$

This contradiction arose from supposing that $G$ has some nonempty anticomponent. Hence, both anticomponents of $G$ are empty; i.e., $G$ is a complete bipartite graph.

Since $G=K_{n_{1}, n_{2}}$, where $n_{2} \geqslant n_{1} \geqslant 1$ and $n=n_{1}+n_{2}$, the average degree of $G$ is equal to $\frac{2 n_{1} n_{2}}{n}$. In virtue of Theorem 3 , the Laplacian eigenvalues of $K_{n_{1}, n_{2}}$ are $n, n_{2}, n_{1}$ and 0 , each with multiplicity $1, n_{1}-1, n_{2}-1$ and 1 , respectively.

Arguing towards a contradiction, suppose that $n_{1} \geqslant 2$. Hence $\mu_{2}(G)=n_{2}$. Since $2 n_{1} \leqslant n$, we deduce that $\bar{d}(G)=\frac{2 n_{1} n_{2}}{n} \leqslant \mu_{2}(G)$, which contradicts the fact that $\sigma(G)=1$. This contradiction proves that $n_{1}=1$ and therefore we conclude that $G=K_{1, n-1}$.

\section{Graphs with $\sigma=1$}

In this section we provide some evidence in order to make plausible Conjecture 1 . We first verify Conjecture 1 for graphs having disconnected complement; namely, we prove that the only graphs having $\sigma=1$ and disconnected complement are the stars (including the trivial star $K_{1}$ ). Then, we prove that Conjecture 1 can be reduced to proving that the only connected and co-connected graph with $\sigma=1$ is $K_{1}$. We then verify Conjecture 1 for cographs, forests, and split graphs.

\subsection{Reduction to co-connected graphs}

We first obtain a result which proves the validity of Conjecture 1 for graphs having disconnected complement.

Corollary 9. Let $G$ be a graph on $n$ vertices such that $\bar{G}$ is disconnected. Then $\sigma(G)=1$ if and only if $G=K_{1, n-1}$. 
Proof. Assume first that $G=K_{1, n-1}$. Then $\bar{d}(G)=2-\frac{2}{n}$. If $n=2$, the Laplacian eigenvalues of $G$ are 2 and 0 . If $n \geqslant 3$, the Laplacian eigenvalues of $G$ are $n, 1$ and 0 , each with multiplicity $1, n-2$ and 1 , respectively. In any case we have that $\sigma(G)=1$.

The 'only if' part follows from Corollary 8.

As a consequence of Corollary 9, Conjecture 1 is equivalent to the validity of the following weaker conjecture.

Conjecture 10. Let $G$ be a graph with connected complement. Then, $\sigma(G)=1$ if and only if $G$ is isomorphic to $K_{1}, K_{2}+s K_{1}$ for some $s>0$, or $K_{1, r}+s K_{1}$ for some $r \geqslant 2$ and $0<s<r-1$.

\subsection{Reduction to connected and co-connected graphs}

We next show that the validity of Conjectures 1 and 10 can be reduced to the validity of the following even weaker conjecture.

Conjecture 11. Let $G$ be a connected graph with connected complement. Then, $\sigma(G)=$ 1 if and only if $G$ is isomorphic to $K_{1}$.

A graph class $\mathcal{G}$ is closed by taking components if every connected component of every graph in $\mathcal{G}$ also belongs to $\mathcal{G}$. In particular, the class of all graphs is closed by taking components. Below we prove that the reduction from Conjecture 1 to Conjecture 11 holds even when restricted to any graph class closed by taking components.

Theorem 12. Let $\mathcal{G}$ be a graph class closed by taking components. If Conjecture 11 holds for $\mathcal{G}$, then Conjecture 1 also holds for $\mathcal{G}$.

Proof. Let $G$ be a graph in $\mathcal{G}$ with $\sigma(G)=1$. Assume first that $G$ is connected. If $G$ is co-connected, by hypothesis, $G$ is isomorphic to $K_{1}$. If $G$ is not co-connected, then $G$ is isomorphic to $K_{1, r}$ for some $r \geqslant 1$, by virtue of Corollary 9 .

Assume now that $G$ is disconnected and let $G=G_{1}+G_{2}$, where each of $G_{1}$ and $G_{2}$ has at least one vertex. We can assume, without loss of generality, that $G_{1}$ is connected and $\mu_{1}\left(G_{1}\right) \geqslant \mu_{1}\left(G_{2}\right)$. If $G_{1}$ were empty, then $G_{2}$ would also be empty, contradicting $\sigma(G)=1$. Hence we can assume, without loss of generality, that $G_{1}$ is nonempty. Let $n_{i}$ and $m_{i}$ denote the number of vertices and edges of $G_{i}$, respectively, for each $i \in\{1,2\}$. Since $\sigma(G)=1$,

$$
\frac{2 m_{2}}{n_{2}} \leqslant \mu_{1}\left(G_{2}\right)<\bar{d}(G)=\frac{2\left(m_{1}+m_{2}\right)}{n_{1}+n_{2}} .
$$

This implies that

$$
\frac{2 m_{2}}{n_{2}}<\frac{2 m_{1}+2 m_{2}}{n_{1}+n_{2}}<\frac{2 m_{1}}{n_{1}} .
$$

As a consequence of (6) we have that

$$
\mu_{2}\left(G_{1}\right)<\frac{2 m_{1}+2 m_{2}}{n_{1}+n_{2}}<\frac{2 m_{1}}{n_{1}}=\bar{d}\left(G_{1}\right) .
$$


We conclude that $\sigma\left(G_{1}\right)=1$. Since $\mathcal{G}$ is closed by taking components, $G_{1} \in \mathcal{G}$. Thus, if $G_{1}$ were co-connected, then $G_{1}=K_{1}$, contradicting the assumption that $G_{1}$ is nonempty. Hence $G_{1}$ is not co-connected and, by Corollary 9 , we have that $G_{1}=K_{1, r}$ for some $r \geqslant 1$.

From (6) we deduce that

$$
\mu_{1}\left(G_{2}\right)<\frac{2 m_{1}+2 m_{2}}{n_{1}+n_{2}}<\frac{2 m_{1}}{n_{1}}=\frac{2 r}{r+1}<2,
$$

and hence, by virtue of Theorem 2 , we conclude that $G_{2}$ must be empty. Then there exists an integer $s \geqslant 1$ such that $G_{2}=s K_{1}$ and therefore it turns out that $G=K_{1, r}+s K_{1}$. The average degree of $G$ is $\bar{d}(G)=\frac{2 r}{r+1+s}$. If $r=1$, then $\sigma(G)=1$ because the second largest Laplacian eigenvalue of $G$ is 0 . If $r \geqslant 2$, then, as the second largest eigenvalue of $G$ is 1 it follows that $\sigma(G)=1$ if and only if $s<r-1$.

A cograph is a graph with no induced $P_{4}$. It is well-known that the only connected and co-connected cograph is $K_{1}[12]$. Hence, Conjecture 11 holds trivially for cographs and, by Theorem 12, Conjecture 1 holds for cographs.

\subsection{Characterizing forests and split graphs with $\sigma=1$}

In this section, we verify Conjecture 1 for forests and split graphs.

A graph class $\mathcal{G}$ is monotone if $G \in \mathcal{G}$ implies that every subgraph of $G$ also belongs to $\mathcal{G}$. Notice that every monotone graph class is closed by taking components. It can be easily seen that the class of all forests is monotone and thus it is closed by taking components.

Theorem 13. Conjecture 1 holds for forests.

Proof. Notice that if $T$ is a connected and co-connected forest, then $T$ is either $K_{1}$ or a tree with diameter greater than two. By virtue of Theorem 12, it suffices to show that if $T$ is a tree with diameter greater than two, then $\sigma(T) \geqslant 2$. Assume that $T$ is a tree with diameter greater than two. Hence there exists two vertices $v_{1}$ and $v_{2}$ such that $d\left(v_{1}\right) \geqslant d\left(v_{2}\right) \geqslant 2>2-\frac{2}{n}=\bar{d}(T)$. By Theorem $2, \mu_{2}(T) \geqslant d_{2}(T) \geqslant 2>\bar{d}(T)$. Therefore, $\sigma(T) \geqslant 2$.

Let $\mathcal{H}$ be a set of graphs. We use the term $\mathcal{H}$-free for referring to the family of those graphs having no graph in $\mathcal{H}$ as induced subgraph. If $\mathcal{H}$ has just one element $H$, we write $H$-free for simplicity. A split graph [4] is a graph whose vertex set can be partitioned into a clique $C$ and a stable set $S$, such a partition $(C, S)$ of its vertices is called a split partition. It is well known that the class of split graphs coincides with the class of $\left\{2 K_{2}, C_{4}, C_{5}\right\}$-free graphs.

Theorem 14. Conjecture 1 holds for split graphs.

Proof. Let $(C, S)$ be a split partition of the graph on $n$ vertices $G$ such that $|C|=c$ and $|S|=n-c$. We label the vertices of $G$ so that $C=\left\{v_{1}, \ldots, v_{c}\right\}$ and $S=\left\{v_{c+1}, \ldots, v_{n}\right\}$. 
We can assume, without loss of generality, that $C$ is a maximal clique of $G$ under inclusion and $d_{i} \geqslant d_{i+1}$, for each $i \in\{1, \ldots, n-1\}$.

We claim that if $G$ is a split graph with $\sigma(G)=1$, then $G$ is isomorphic to $K_{1, r-1}+$ $(n-r) K_{1}$ for some $r$ such that $2 \leqslant r \leqslant n$.

In order to prove our claim we assume that $G$ is nonisomorphic to $K_{1, r-1}+(n-r) K_{1}$, for each $r \in\{2, \ldots, n\}$ and we will prove that $\sigma(G) \geqslant 2$. By virtue of Theorem 2, it suffices to prove that $d_{2} \geqslant \bar{d}(G)$ or equivalently that

$$
\sum_{i=3}^{n}\left(d_{2}-d_{i}\right) \geqslant d_{1}-d_{2} .
$$

We will consider two cases.

1. Assume that $d_{2} \geqslant c$. Since $C$ is a maximal clique, $d_{2}-d_{i} \geqslant 1$ for each $i \in$ $\{c+1, \ldots, n\}$. Hence

$$
\sum_{i=3}^{n}\left(d_{2}-d_{i}\right) \geqslant \sum_{i=c+1}^{n}\left(d_{2}-d_{i}\right) \geqslant n-c \geqslant d_{1}-d_{2} .
$$

2. Assume that $d_{2}=c-1$. Our assumption on $G$ implies that $c>2$. Moreover, we have that $d_{i} \leqslant 1$ for each $i \in\{c+1, \ldots, n\}$. Consequently, $d_{2}-d_{i} \geqslant 1$ for each such $i$ and the reasoning follows as above.

Thus we have proved our claim. In particular, the only connected and co-connected split graph with $\sigma=1$ is $K_{1}$; i.e., Conjecture 11 holds for split graphs. Therefore, by virtue of Theorem 12, Conjecture 1 holds for split graphs.

\section{Acknowledgements}

Authors would like to thank the anonymous referees for generous observations and comments that we have made ample use of in the paper. A. Cafure and E. Dratman acknowledge partial support of CONICET PIP 112-2013-010-0598. A. Cafure, E. Dratman, L. N. Grippo, and M. D. Safe acknowledge partial support of CONICET UNGS-14420140100027-CO. L. N. Grippo and M. D. Safe acknowledge partial support of UNS Grant PGI 24/L103. L. E. Allem, M. D. Safe and V. Trevisan acknowledge partial support of MATH-AmSud 18-MATH-01. V. Trevisan acknowledges partial support of CNPq grants 409746/2016-9 and 303334/2016-9 and FAPERGS (Proj. PqG 17/2551-0001).

\section{References}

[1] Brouwer, A. E. and Haemers, W. H., A lower bound for the Laplacian eigenvalues of a graph-proof of a conjecture by Guo, Linear Algebra Appl. 429 (2008), pp. 21312135. 
[2] Das, K. C., S. A. Mojallal and I. Gutman, On Laplacian energy in terms of graph invariants, Appl. Math. Comput. 268 (2015), pp. 83-92.

[3] Das, K. C., S. A. Mojallal and V. Trevisan, Distribution of Laplacian eigenvalues of graphs, Linear Algebra Appl. 508 (2016), pp. 48-61.

[4] Földes, S. and P. L. Hammer, Split graphs, in: Proceedings of the Eighth Southeastern Conference on Combinatorics, Graph Theory and Computing (Louisiana State Univ., Baton Rouge, La., 1977), Utilitas Math., Winnipeg, Man., 1977 pp. 311-315.

[5] Grone, R. and R. Merris, The Laplacian spectrum of a graph II, SIAM J. Discrete Math. 7 (1994), pp. 221-229.

[6] Guo, J.-M., On the third largest Laplacian eigenvalue of a graph, Linear Multilinear Algebra 55 (2007), pp. 93-102.

[7] Li, J.-S. and Y.-L. Pan, A note on the second largest eigenvalue of the Laplacian matrix of a graph, Linear and Multilinear Algebra 48 (2000), pp. 117-121.

[8] Merris, R., Laplacian matrices of graphs: a survey, Linear Algebra Appl. 197/198 (1994), pp. 143-176.

[9] Mohar, B., The Laplacian spectrum of graphs, in: Graph theory, combinatorics, and applications. Vol. 2 (Kalamazoo, MI, 1988), Wiley-Intersci. Publ., Wiley, New York, 1991 pp. 871-898.

[10] Mohar, B., Laplace eigenvalues of graphs-a survey, Discrete Math. 109 (1992), pp. 171-183.

[11] Pirzada, S. and H. A. Ganie, On the Laplacian eigenvalues of a graph and Laplacian energy, Linear Algebra Appl. 486 (2015), pp. 454-468.

[12] Seinsche, D., On a property of the class of $n$-colorable graphs, J. Combinatorial Theory Ser. B, 16 (1974), pp. 191-193.

[13] West, D. B., "Introduction to Graph Theory," Prentice Hall, 2000, 2nd edition. 